\title{
USO Y APROVECHAMIENTO DE LA FAUNA SILVESTRE EN LA SELVA ZOQUE, MÉXICO
}

\author{
IVÁN LIRA-TORRES, MiguEL BRIONES-SALAS, ${ }^{2}$ FABIÁN RiCARdo GÓMEZ \\ DE ANDA, ${ }^{1}$ Deyanira OJEDA-RAMÍREZ ${ }^{1} \&$ ARMando PELÁEZ ACERO ${ }^{1}$ \\ ${ }^{1}$ Instituto de Ciencias Agropecuarias, Universidad Autónoma del Estado de Hidalgo, Área Académica \\ de Medicina Veterinaria y Zootecnia. Rancho Universitario Av. Universidad km 1, Ex-Hda. de \\ Aquetzalpa A.P. 32 Tulancingo, Hgo. México. CP.43600.<ilira_12@hotmail.com> \\ ${ }^{2}$ Centro Interdisciplinario de Investigación para el Desarrollo Integral Regional, Unidad Oaxaca \\ (CIIDIR-OAX.), IPN. Hornos 1003, Santa Cruz Xoxocotlán, Oaxaca, Oax., C.P.71230; \\ $<$ mbriones@ipn.mx>
}

Lira-Torres, I., Briones-Salas, M., Gómez de Anda, F. R., Ojeda-Ramírez, D. \& Peláez A., A. 2014. Uso y Aprovechamiento de Fauna Silvestre en la Selva Zoque, México. Acta Zoológica Mexicana (n.s.), 30(1): 74-90.

RESUMEN. La cacería de subsistencia y la pérdida de hábitat son dos de las principales actividades humanas que tienen un efecto negativo sobre la fauna silvestre en las fronteras de colonización. En la presente investigación se estudió el uso consuntivo que se da a la fauna silvestre en varias comunidades de la Selva Zoque, México. Durante el año 2012 y principios del 2013 se realizó una serie de visitas a la región para conocer y listar la riqueza de especies y usos que los habitantes hacen de ella, describir los métodos de caza más utilizados, las especies preferidas y su frecuencia y/o aprovechamiento. En total se cazaron 202 animales por 13 cazadores participantes, el grupo biológico más utilizado es el de los mamíferos, $N=13$ especies; las aves el segundo grupo, $N=4$ especies y los reptiles el menos aprovechado, $N=1$ especies. El aprovechamiento de esta última clase está orientado al uso alimenticio, y en menor grado, al artesanal, a diferencia de los otros dos grupos, que en su mayoría tienen uso alimentario, control, ornamental y medicinal. El total de biomasa obtenida durante el estudio fue de $1900 \mathrm{~kg}$. La biomasa aprovechada varió significativamente entre especies $(p>0.001)$. La mayor producción de carne se obtuvo a partir de: Pecari tajacu, Cuniculus paca, Dasypus novemcinctus, Tapirus bairdii, Mazama temama y Tayassu pecari. La batida fue la técnica más usada, también se identificaron las técnicas de espía o acecho en sitios de alimentación, parcelas de trabajo y lampareo en áreas conservadas. Los tipos de armas más empleadas fueron las escopetas calibre $0.12,0.16,0.20$ y rifle 0.22 . La cacería de subsistencia es una actividad complementaria a las actividades agrícolas y ganaderas que las poblaciones campesinas realizan en la Selva Zoque; sin embargo, es necesario continuar realizando monitoreos para planear la sustentabilidad de ese recurso.

Palabras clave: Aprovechamiento, Fauna Silvestre, Selva Zoque, Sustentabilidad, México.

Recibido: 03/04/2013; aceptado: 02/12/2013. 
Lira-Torres, I., Briones-Salas, M., Gómez de Anda, F. R., Ojeda-Ramírez, D. \& Peláez A., A. 2014. Use and development of hunting wildlife at Zoque forest, Mexico. Acta Zoológica Mexicana (n.s.), 30(1): 74-90.

ABSTRACT. Subsistence hunting and habitat loss are two of the main human activities that have a negative effect on wildlife border settlement. In the present study, it was met the assumption of the great value and different uses that is given to wildlife in several communities of the Zoque Forest located in Mexico. During 2012 and early 2013 were made some visits to the region, to find and list the species richness, and uses that people make of them; describe the methods used, the preferred species and their frequency of hunting and harvesting of animals. A total of 202 animals were hunted by 13 participants hunters, mammals is the biological group most used, $N=13$ species, birds are the second group, $N=4$ species, and the less exploited is the reptiles group, $N=1$ species. The exploitation of the reptiles group is pointed to food use, and in less rank in handicrafts, unlike the mammals and birds, which has a food, plague control, ornamental and medicinal use. The total animal biomass obtained during the study was $1900 \mathrm{~kg}$. Total biomass harvested varied significantly among species $(p>0.001)$. The majority production of meat was obtained from: Pecari tajacu, Cuniculus paca, Dasypus novemcinctus, Tapirus bairdii, Mazama temama y Tayassu pecari. Herding was the most widely used technique for capturing animals; there was also employed lurking in the animal's feeding sites, as well as in the population's places of work. The types of calibers in the weapons more used were $0.12,0.16,0.20$ in shotguns and 0.22 in rifles. Subsistence hunting is a complementary activity to the farming and stockbreeding activities that rural populations in the Zoque Forest do. However, we need to continue performing monitoring sustainability of hunting.

Key words: Exploitation, Mexico, Tropical rain forest, Sustainability, Zoque Forest, Wildlife.

\section{INTRODUCCIÓN}

La fauna silvestre de la Selva Zoque; eco-región que incluye los Chimalapa en Oaxaca, los Uxpanapa en Veracruz y la Reserva de la Biosfera Selva El Ocote en Chiapas, constituye un recurso natural para los habitantes locales. Numerosas especies de mamíferos, aves y reptiles han sido y siguen siendo aprovechadas con fines de alimento, vestimenta, medicina tradicional, herramientas, objetos rituales, símbolos, trofeos, y mascotas (Shaw 1991, Robinson \& Bennett 2000, González-Pérez et al. 2004, Ortega del Valle et al. 2012).

La importancia nutricional, económica y social de la fauna para los habitantes de la Selva Zoque y del Sureste de México ha sido escasamente evaluada en términos cuantitativos, a pesar de que numerosas manifestaciones culturales como la gastronomía, la danza y las artes plásticas evidencian la relevancia de los animales silvestres en la región (Escamilla et al. 2000, Cid 2001, Quijano-Hernández \& Calmé 2002, Naranjo et al. 2004, Reyna-Hurtado \& Tanner 2007, Lira-Torres \& Briones-Salas 2011, Lira et al. 2012). Gran parte de estas selvas y bosques han sido sometidos a un intenso uso agropecuario desde el inicio de la época colonial hasta nuestros días (CONABIO 2009). Muchas de estas áreas presentan una alta densidad humana, cuyas actividades económicas han provocado una elevada tasa de fragmentación y pérdida de los bosques tropicales húmedos, secos y mesófilos que originalmente los cubrían (Gobierno del estado de Oaxaca 1990, Laurance \& Bierregaard 1997, Caballero 2000, Lira-To- 
rres \& Briones-Salas 2012). Si bien la ganadería extensiva de doble propósito: carne y leche, la avicultura, la porcicultura, así como los cultivos de maíz, fríjol, papaya, cacahuate, melón, naranja, sandia y extracción de hule, son actividades predominantes en la región, el uso de la fauna silvestre ha representado una fuente complementaria de alimento para sus pobladores (Aparicio 2001, Ortega del Valle et al. 2012).

Desafortunadamente, las prácticas de cacería no sustentables y la destrucción y fragmentación de las selvas tropicales han originado cambios importantes en la distribución y la abundancia de numerosas poblaciones de fauna silvestre (Caballero 2000, Cid 2001, Naranjo et al. 2004). No todas las especies han sido igualmente afectadas, por un lado, el marín o pecarí de labios blancos (Tayassu pecari), el jaguar (Panthera onca), la guacamaya roja (Ara macao), el loro cabeza azul (Amazona farinosa), el tapir centroamericano (Tapirus bairdii), el mono araña (Ateles geoffroyi) y el saraguato o mono aullador (Alouatta palliata) han sufrido una severa declinación y aislamiento en sus poblaciones locales (Lira et al. 2012); mientras que especies como la zorra gris (Urocyon cinereoargenteus), la chachalaca (Ortalis sp.), la iguana negra (Ctenosaura pectinata), el jabalí de collar (Tayassu pecari) y el coyote (Canis latrans) han sido poco o nada afectadas por la transformación de los bosques maduros en sistemas agropecuarios y áreas de vegetación secundarias (Robinson \& Bennett, 2000, Lira 2006).

El presente estudio analiza el uso consuntivo, el cual se define como el valor directo a la fauna silvestre; tales como cazar, extraer, capturar y/o utilizar sus derivados, por las distintas comunidades de la zona norte del municipio de Santa María Chimalapas, Oaxaca, así como en la Región de los Uxpanapa, Veracruz, en la Selva Zoque, y que afecta de manera directa a este recurso (Ojasti 2000). Así como en describir los métodos utilizados para la captura de los animales silvestres, las especies preferidas y su frecuencia de caza o aprovechamiento.

\section{MATERIALES Y MÉTODOS}

Área de Estudio. La Selva Zoque, se encuentra localizada en los municipios, congregaciones y ejidos: a) Oaxaca: Cabecera Municipal de Santa María Chimalapa y San Miguel Chimalapa, Congregación de la Fortaleza y San Francisco La Paz, Ejidos La Esmeralda, y El Porvenir, Región de Nizanda, que abarca los Municipios de La Venta, La Ventosa, Santiago Laollaga, Santo Domingo Ingenio; b) Veracruz: Uxpanapa, Ejido de Tamayo y Poblados 10, 11, 12, 13, 14 y 15; c) Chiapas: Reserva de la Biosfera Selva El Ocote y Rancho Los Ocotones, Cintalapa de Figueroa; coordenadas $16^{\circ} 00^{\prime} 32^{\prime \prime}$ a $17^{\circ} 32^{\prime} 00^{\prime \prime} \mathrm{N}-93^{\circ} 21^{\prime} 40^{\prime \prime}$ a $94^{\circ} 53^{\prime}$ 53' $\mathrm{W}$ (Fig. 1). El clima predominante es cálido húmedo con lluvias en verano; $\mathrm{Am}(\mathrm{f})$ y $(\mathrm{A}) \mathrm{C}(\mathrm{w} 2)(\mathrm{García}$ 1973). La precipitación y temperatura varían de 1000 a $1800 \mathrm{~mm}$ y de $18{ }^{\circ} \mathrm{C}$ a $22^{\circ} \mathrm{C}$ respectivamente (Arriaga et al. 2000) (Fig. 1). 


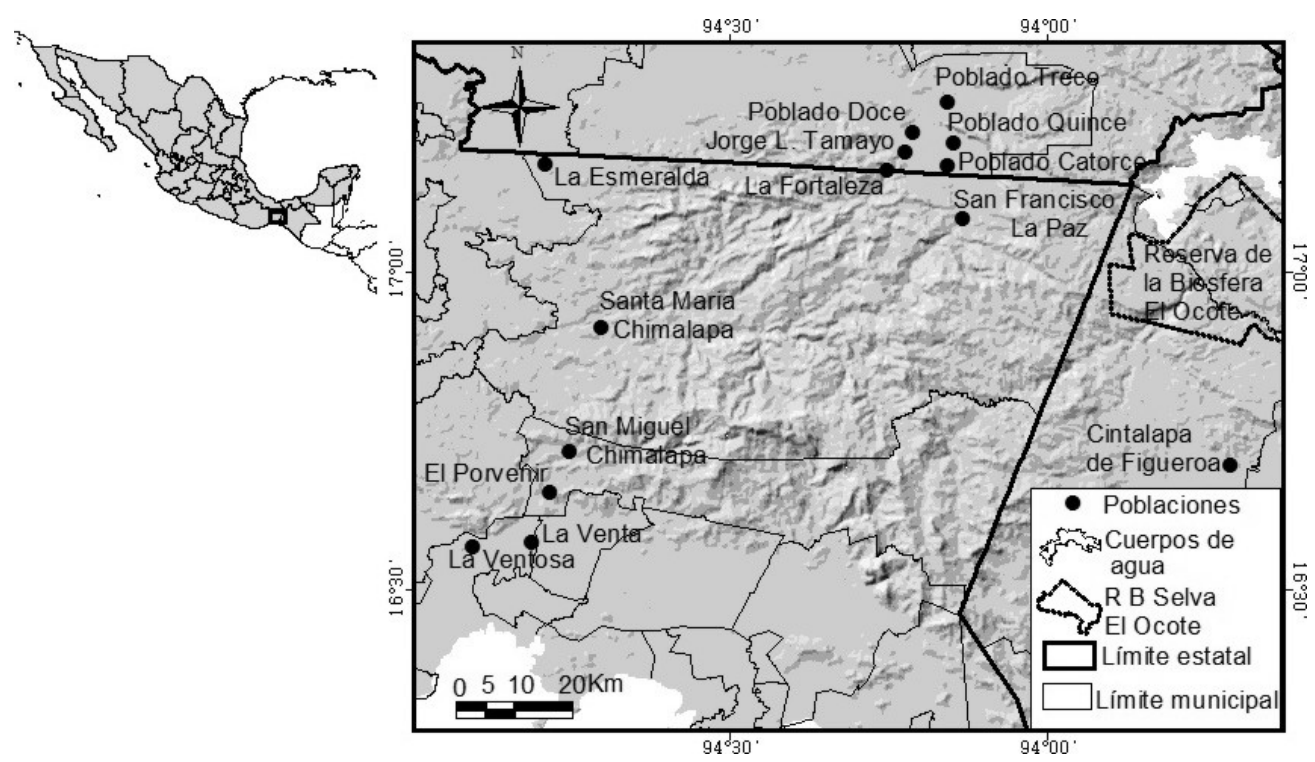

Figura 1. Ubicación geográfica del Área de Estudio, principales ejidos muestreados.

La Selva Zoque está compuesta por diferentes tipos de vegetación: bosque tropical perennifolio, subperennifolio y caducifolio, bosque mesófilo de montaña, bosques de coníferas y sabanas (Rzedowski 1991). De la superficie total, el 78.3\% corresponde a vegetación bien conservada en la que el bosque tropical perennifolio cubre aproximadamente el $48 \%$, seguida del bosque tropical subperennifolio $14.4 \%$ y bosque mesófilo de montaña 13.5\% (Salas et al. 2001, González 2004).

Trabajo de campo. Se realizaron salidas de campo durante el año 2012 y principio del 2013 como parte del proyecto: Evaluación de la Ganadería Extensiva y Cacería de Subsistencia sobre las poblaciones de Carnívoros y sus Presas, que se realizan en la zona norte del municipio de Santa María Chimalapa, Oaxaca, así como del municipio de Uxpanapa, Veracruz, financiado por el Fondo Mundial para la Naturaleza (WWF) / Alianza Fundación Carlos Slim y la Fundación Comunitaria Oaxaca A.C., en la Selva Zoque. Debido a solicitud de los financiadores, la Reserva de la Biosfera Selva El Ocote, en el Estado de Chiapas; y perteneciente a la eco-región Selva Zoque, quedo fuera del muestreo solicitado.

De acuerdo a los criterios de Furze et al. (1996) se realizó un listado preliminar de informantes clave con experiencia en la región (cazadores). Esta selección se realizó mediante un muestreo no aleatorio, con base en las recomendaciones de las autoridades, familiares y líderes, sobre personas que practicaban la cacería con mayor frecuencia. Es importante mencionar que desde el inicio del estudio, los cazadores 
fueron informados acerca de los objetivos de la investigación (corto, mediano y largo plazo), así como de su importancia; así mismo, ellos dieron el consentimiento para usar la información que proporcionarían, con la finalidad de caracterizar la cacería y el uso de la fauna silvestre en la región. Todos mostraron interés en el proceso planteado y accedieron a colaborar para alcanzar los objetivos propuestos. Adicionalmente, el grupo de trabajo se involucró en esta actividad durante las visitas de prospección, esto debido a la confianza que se ha logrado a lo largo de 10 años de trabajo en la región. Esta actividad fue sumamente importante porque se les enseñó a llevar los registros de caza adecuadamente para obtener mejores registros de la cacería, o de la captura de especies protegidas, lo cual resultó en una interpretación más apropiada y real de los datos obtenidos.

Del total de entrevistados (120 personas), 13 cazadores aceptaron llevar un registro anual de las actividades de caza que realizan en la Selva Zoque en el 2012 y principios del 2013. Los cazadores participantes pertenecieron a las localidades de: La Congregación de La Fortaleza, Municipio de Santa María Chimalapa, Oaxaca y cazadores de los poblados 10,11, 12, 13, 14 y 15 del Municipio de Uxpanapa, Veracruz (Fig. 1). La edad promedio de los hombres, quienes son los principales actores, era entre los 18 a los 60 años, dedicándose la mayoría a actividades relacionadas con la ganadería, agricultura, la caza y pesca. El núcleo familiar de los participantes osciló entre una y siete personas. Por ética profesional y para evitar represalias por parte de la Procuraduría Federal de Protección al Ambiente (PROFEPA) del Gobierno Federal, la identidad y dirección de los colaboradores se mantuvieron en el anonimato.

A todas las personas involucradas se les proporcionó una libreta de campo para que registraran los eventos de cacería, en esta libreta el cazador anotó: fecha, hora de inicio y fin de la caza, nombre, edad y número de cazadores participantes, tipo de técnica de cacería, número de perros, arma utilizada, animales cazados, sexo, peso y tipo de vegetación donde se cazaron, datos etnozoológicos y de uso. Con los registros obtenidos se identificaron todas las especies cazadas y el número de individuos, se calculó la biomasa animal obtenida por especie y se hicieron comparativos entre clases de vertebrados mediante una prueba de análisis de varianza (ANOVA Spiegel et al. 2007). Finalmente se determinó la técnica de caza más utilizada y la biomasa promedio obtenida anualmente (Robinson \& Redford 1991, Naranjo et al. 2004, Bello \& Reyna 2010).

\section{RESULTADOS}

Especies y número de individuos cazados. En total se cazaron 202 animales por los 13 cazadores participantes, el grupo biológico con más especies utilizadas fue el de los mamíferos con 13 especies, las aves fueron el segundo grupo con 4 especies, y los reptiles el grupo menos aprovechado con una especie (Cuadro 1). 


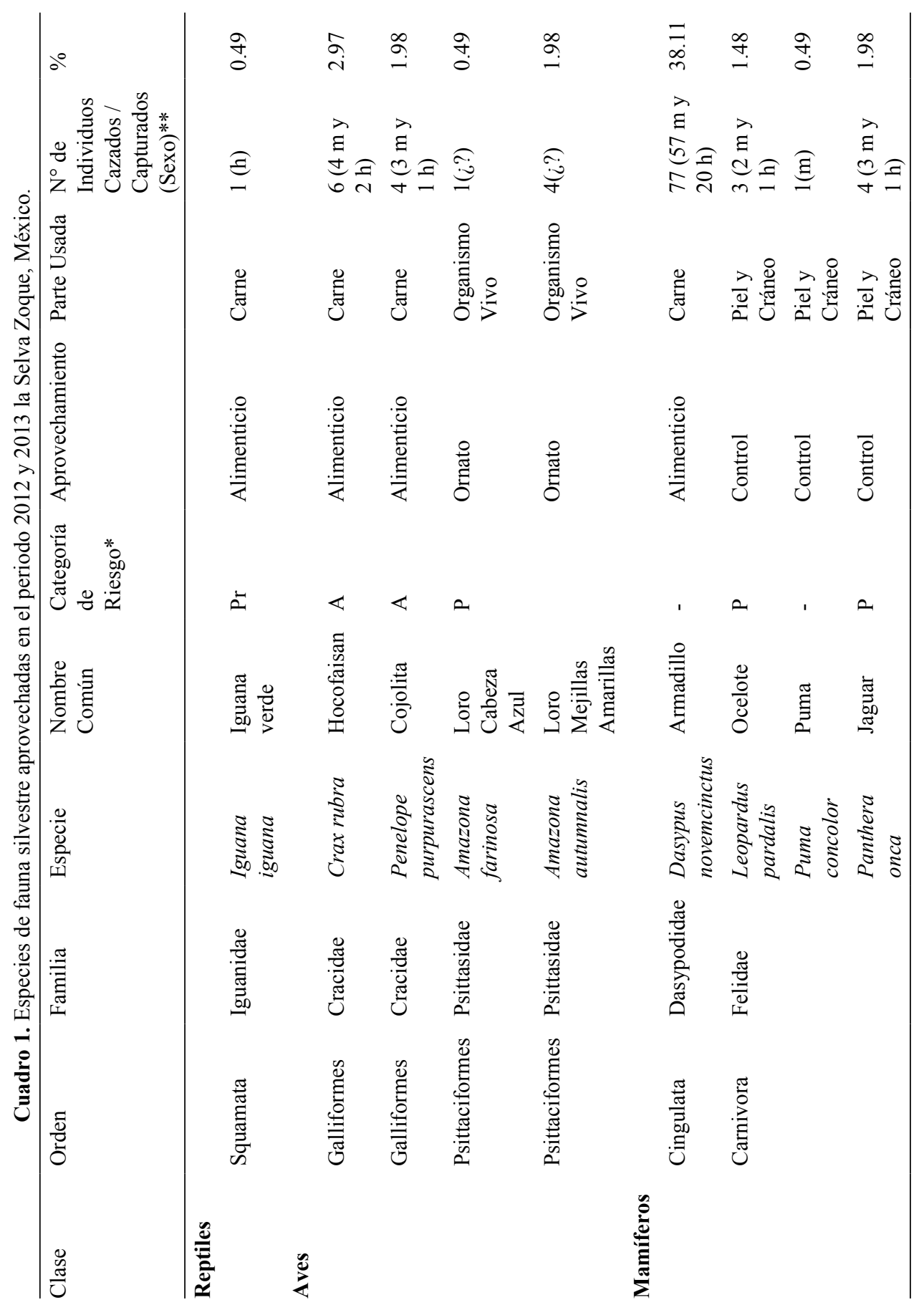




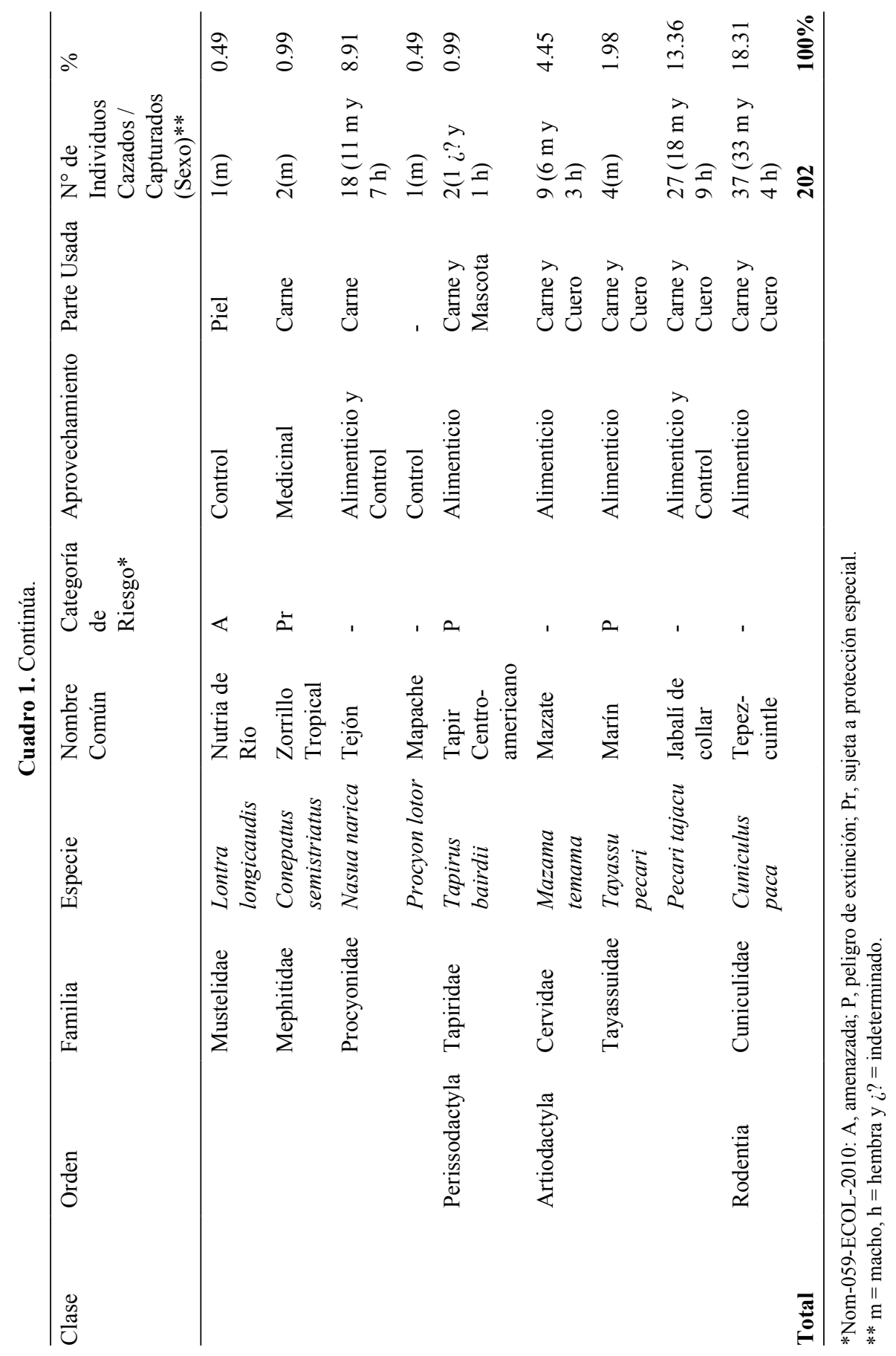


Las especies de mamíferos más usadas para la alimentación y/o control de daños fueron: el armadillo (Dasypus novemcintus), $\operatorname{con} N=77$; el Tepezcuintle (Cuniculus paca), con $N=37$; el Jabalí de Collar (Pecari tajacu), con $N=27$; el Tejón (Nasua narica), $N=18$; el Venado Mazate (Mazama temama), $N=9$; el Marín (Tayassu pecari), $N=4$; el Zorrillo Tropical (Conepatus semistriatus), $N=2$; un Tapir Centroamericano (Tapirus bairdii), $N=1$ y un Mapache (Procyon lotor), $N=1$.

Las especies de mamíferos que fueron afectadas como control de daños para prevenir y/o resolver ataques hacia el ganado domestico (bovinos y equinos) fueron: Jaguar (Panthera onca), $N=4$; Puma (Puma concolor), $N=1$; Ocelote (Leopardus pardalis), $N=2$ y una Nutria de Río (Lontra longicaudis) juvenil, $N=1$.

Las especies de mamíferos que se mantienen como mascotas (ornamental) por pobladores de las comunidades son: Ocelote (Leopardus pardalis), $N=1 \mathrm{y}$ un Tapir Centroamericano (Tapirus bairdii) juvenil, $N=1$.

Con respecto a las aves, las que se aprovecharon para la alimentación fueron el Hocofaisan (Crax rubra), $N=6$ y la Cojolita (Penelope purpurascens), $N=4$. Con respecto a la posesión de aves de ornato se observaron el Loro Mejillas Amarillas (Amazona autumnalis), $N=4$ y de Loro Cabeza Azul (Amazona farinosa), $N=1$, ambas especies de sexo indeterminado. Finalmente, solamente se observo en una familia el aprovechamiento para alimentación de una Iguana verde hembra (Iguana iguana), $N=1$.

Biomasa y peso de individuos con mayor presión de cacería. El total de biomasa animal obtenida en este estudio durante 2012 y principios del 2013 fue de 1,900 kg, de la cual el $66.66 \%$ se obtuvo de 12 especies, que fueron las que tuvieron mayores registros de caza. La biomasa total aprovechada varió significativamente entre especies $(P>0.001)$. La mayor producción de carne se obtuvo a partir seis especies de mamíferos, estas son: Pecari tajacu (540 kg), Cuniculus paca (444 kg), Dasypus novemcinctus (308 kg), Tapirus bairdii (200 kg), Mazama temama $(162 \mathrm{~kg})$ y Tayassu pecari $(120 \mathrm{~kg})$. Finalmente, Crax rubra fue la ave más aprovechada en la región (30 $\mathrm{kg}$ ) (Cuadro 2).

Formas de uso. Los tipos de uso de la fauna silvestre registrados en orden de importancia en este estudio son: a) alimentario, caracterizado por la ingesta de carne de monte y control de daños en los cultivos; b) control, para la protección al evitar la depredación de su ganado por grandes felinos; c) ornamental, al tener a estos animales silvestres como mascotas o animales de traspatio; y d) medicinal, cuando se utiliza a los animales con fines curativos (Cuadro 3).

Técnicas de cacería utilizadas, perros y armas empleadas. La batida en áreas de vegetación secundaria (acahuales) fue la técnica más usada al realizar esta investigación; también se identificaron otras como la espía o el acecho en sitios de alimentación y parcelas de trabajo, así como lampareo en áreas conservadas. Por otro lado, los perros son comúnmente empleados durante las batidas, el número promedio de 


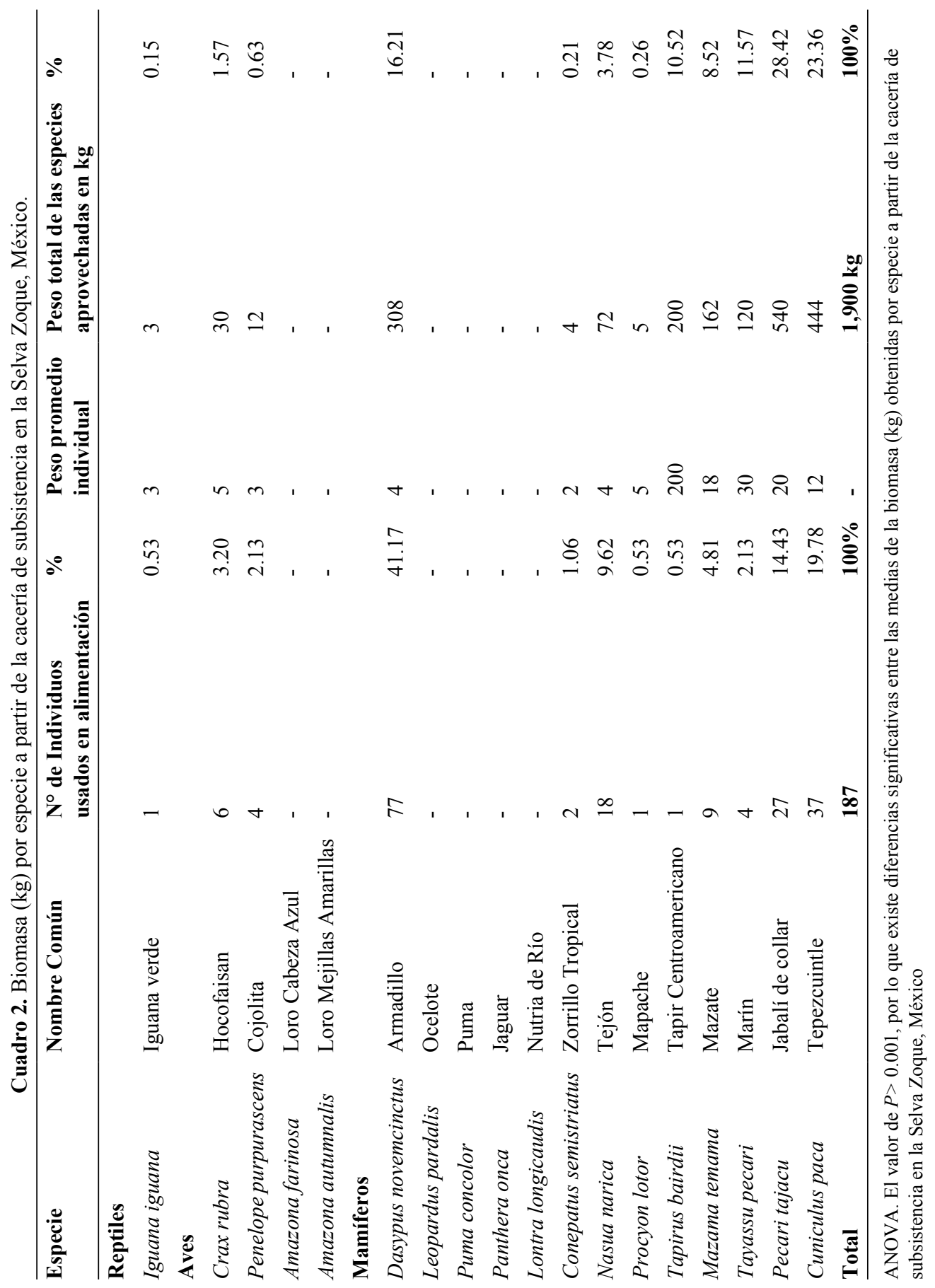


Cuadro 3. Motivaciones para el uso de fauna por cazadores participantes.

\begin{tabular}{lcccccc}
\hline & \multicolumn{3}{c}{ Motivación } & & & \\
\hline $\begin{array}{l}\text { Cazador y Región de } \\
\text { Procedencia }\end{array}$ & Alimento & Control & $\begin{array}{c}\text { Alimento } \\
\text { medicinal }\end{array}$ & $\begin{array}{c}\text { Alimento } \\
\text { control }\end{array}$ & Ornato & Total \\
\hline Chano / Chimalapas, Oaxaca & 1 & - & - & - & 1 & 2 \\
Chato / Chimalapas, Oaxaca & 1 & 1 & 1 & 1 & - & 4 \\
Abel / Chimalapas, Oaxaca & 1 & 1 & - & 1 & - & 3 \\
Delfino / Chimalapas, Oaxaca & 1 & 1 & - & 1 & 1 & 4 \\
Felipe / Chimalapas, Oaxaca & 1 & 1 & - & 1 & - & 3 \\
Galeana / Chimalapas, Oaxaca & 1 & 1 & - & 1 & 1 & 4 \\
Ernesto / Chimalapas, Oaxaca & 1 & 1 & - & 1 & - & 3 \\
Rogelio /Chimalapas, Oaxaca & 1 & 1 & - & 1 & 1 & 4 \\
Juan / Uxpanapa, Veracruz & - & 1 & - & - & - & 1 \\
Francisco / Uxpanapa, Veracruz & 1 & 1 & - & 1 & - & 3 \\
Pablo / Uxpanapa, Veracruz & 1 & - & - & 1 & - & 2 \\
Pedro / Uxpanapa, Veracruz & 1 & 1 & - & 1 & - & 3 \\
Pablo Prieto / Uxpanapa, Veracruz & - & 1 & - & - & 1 & 2 \\
Total & $\mathbf{1 1}$ & $\mathbf{1 1}$ & $\mathbf{1}$ & $\mathbf{1 0}$ & $\mathbf{5}$ & $\mathbf{3 8}$ \\
\hline
\end{tabular}

animales utilizados fue de ocho individuos. Los tipos de arma más empleadas fueron la escopeta calibre 0.12 y 0.16 de un tiro (72\%) seguida de los calibres 0.20 y rifle 0.22 (26.4 y $1.6 \%$ respectivamente).

\section{DISCUSIÓN}

El análisis de nuestros resultados muestra similitudes y diferencias con otros estudios realizados en diferentes localidades en el Neotrópico. En general, la fauna silvestre constituye un recurso que es utilizado de diversas maneras e intensidades, dependiendo de las condiciones y necesidades locales (Escamilla et al. 2000, Naranjo et al. 2004, Guerra et al. 2010, Bernal 2011).

En el Neotrópico la cacería ha sido generalmente estudiada mediante entrevistas personales a cazadores y habitantes locales (Naranjo et al. 2004, Guerra et al. 2010). Para el caso de la Selva Zoque, la caracterización de la cacería de subsistencia constituye una de las bases para el manejo comunitario del recurso en el área. Por ello, es muy importante desarrollar metodologías complementarias para promover la participación de los habitantes locales desde el inicio del proceso. En el presente estudio, los cazadores fueron capacitados como técnicos de campo y toda la información incluida en este documento se basa en sus valiosos aportes. Las visitas mensuales y entrevistas personales propiciaron espacios de articulación que fortalecieron los vínculos de 
confianza entre los pobladores de las diferentes comunidades y los investigadores, así como la participación conjunta en el proceso planteado. La metodología utilizada también permitió extender la fase de caracterización a mayor escala temporal y espacial. El contacto estrecho con los habitantes involucrados y la realización de evaluaciones participativas del proceso contribuyó a incrementar la confiabilidad de las tendencias de caza estimadas.

Especies y número de individuos cazados. Con respecto a las causas de su uso frecuente en la localidad de estudio, podemos decir que las causas que llevan a la cacería de las especies citadas en este estudio son: 1) la mayoría de los animales cazados son de hábitos diurnos a excepción de $D$. novemcinctus y $C$. paca, característica que es aprovechada por la mayoría de los cazadores, quienes practican esta actividad de manera "oportunista" cuando encuentran la presa en el camino a sus parcelas o potreros; 2) T. tajacu, $N$. narica y $P$. lotor, son las especies que más afectan a los cultivos de maíz, fríjol, calabaza y otros, por lo que a través de su caza además de obtener carne para el hogar funciona como un método de control de daños; 3 ) los usos y costumbres tradicionales así como la palatabilidad de algunas carnes, como es el caso de C. paca, M. temama, T. pecari y C. rubra; y 4) la protección del ganado domestico, bovino, ovino, porcino y perros domésticos, de la depredación principalmente de $P$. onca y $P$. concolor, que comúnmente se acercan a las comunidades a buscar presas alternativas (Castro-Franco \& Bustos 2003, González-Pérez et al. 2004, Lira et al. 2012). Vale la pena recalcar que son los mismos comuneros quienes han decidido solo sacrifican grandes felinos que reinciden en la depredación de ganado para no afectar a las poblaciones de estas especies, debido a que se ha hecho trabajo de concientización y educación ambiental con ellos en años anteriores (Lira-Torres \& Ramos-Fernández 2007).

Hay que considerar que la caza de animales para la alimentación es de forma oportunista y no selectiva, es decir, aunque existen preferencias, los cazadores de subsistencia tienden a encontrar a sus presas casualmente durante su faena diaria. Por lo tanto, los animales pueden ser cazados simplemente porque se "les atraviesan" y no como consecuencia de una búsqueda deliberada. Asimismo, es importante considerar que la frecuencia y preferencia de utilización de fauna puede diferir de un cazador a otro debido a factores como la abundancia de la especie, la accesibilidad para cazarla y la habilidad inherente de cada cazador para obtener un ejemplar. A lo anterior, se agregan factores conductuales como sistemas de creencias que favorecen o desfavorecen la cacería de ciertas especies, como tabúes y supersticiones, preferencias alimentarias, percepciones sobre la abundancia o la rareza de los animales, e incluso acciones de conservación (Winter 2002).

Biomasa y peso de individuos con mayor presión de cacería. En los mercados regionales de las cabeceras municipales, ejidos y congregaciones de la Selva Zoque, el comercio y la cacería de subsistencia de fauna está permitida extraoficialmente para 
los campesinos por las autoridades correspondientes, con la finalidad de que obtengan de esta forma los productos de origen animal y recursos económicos que requieren (Cid 2001, Bodmer \& Robinson 2004, González-Pérez et al. 2004, Naranjo et al. 2004, Lira et al. 2012). Lo anterior, se sustenta por la extracción de carne de monte durante el año 2012 y principios del 2013 por 13 familias de la región, la cual fue de $1,900 \mathrm{~kg}$, sin considerar las familias de otras congregaciones o ejidos que no quisieron participar, lo anterior seguramente tiene un efecto negativo sobre las poblaciones de grandes y medianos mamíferos y aves silvestres (Naranjo et al. 2004, Reyna-Hurtado \& Tanner 2007, Lira-Torres \& Briones-Salas 2011, Lira et al. 2012).

No obstante, no es posible realizar extrapolaciones de las tendencias de caza observadas con el resto de las comunidades de la región, ya que se desconoce el número de cazadores existentes en la zona. Este parámetro es difícil de estimar porque cualquier hombre entre los 18 y los 60 años de edad es un cazador "potencial", cuyos patrones de caza varían individualmente. Además la cacería es dinámica en el tiempo y espacio, por lo cual es difícil estimar un tamaño de muestra que permita capturar la variabilidad existente (Ayres 1991). Sin embargo, si a estas condiciones se suma el mercadeo al menudeo para satisfacer necesidades económicas mínimas de las familias campesinas y el gran mercado de mayoreo de animales vivos y productos, se podría obtener un resultado que se estimaría en varios miles de animales vivos y algunas toneladas de productos que mensualmente son extraídos de las selvas y bosques de la Selva Zoque.

Aunque se tienen indicios de que los parámetros poblacionales de las especies cazadas están aparentemente estables (Lira-Torres \& Briones-Salas 2012), debido a que la mayor parte de la cacería en esta región se practica en zonas de vegetación secundaria (Acahuales), cercanas a hábitat poco perturbados, mismos que actúan como fuente y sumidero; es importante hacer un monitoreo en áreas donde esta actividad sea heterogénea para evitar que los datos de producción pudieran estar sesgados (Bodmer \& Robinson 2004). Así mismo, es importante llevar a cabo trabajos de investigación que permitan crear modelos predictivos que incorporen el conocimiento tradicional que los comuneros poseen para planear su uso racional. Se requiere que las autoridades ambientales propongan formas alternativas de uso y aprovechamiento de la fauna silvestre, porque las UMA'S y los programas productivos implementados en la región; apicultura y huertos familiares, por parte del Gobierno Federal a través de la Comisión Nacional de Áreas Naturales Protegidas (CONANP) no han tenido un buen desarrollo en esta región.

Formas de uso. Los principales usos que se le da a la fauna silvestre presente en la región se documentan el Cuadro 3. Todos los entrevistados aceptaron su gusto por la carne de monte que constituye una de las principales fuentes de proteína animal en la región (González-Pérez et al. 2004).

De los animales mantenidos como mascotas, existe una subestimación en el nú- 
mero; principalmente de loros y pericos (Amazona sp y Aratinga $s p$ ), ya que este trabajo solo registró la presencia de estas especies en las familias de los cazadores participantes, pero se observó que muy frecuentemente en cualquier casa de la región se tenía en posesión un ejemplar o más de estas especies. Al respecto la Ley General de Vida Silvestre; artículo 60 Bis 2, dice: Ningún ejemplar de ave correspondiente a la familia Psittacidae o psitácido, cuya distribución natural sea dentro del territorio nacional, podrá ser sujeto de aprovechamiento extractivo con fines de subsistencia o comerciales. No obstante, la mayoría de los residentes de las comunidades desconocen la normatividad vigente en cuanto al aprovechamiento de recursos naturales, dado que su máximo grado de estudio es la educación inicial (Primaria) y por otro lado, son pocas las campañas de concientización y protección de los recursos naturales que se realizan en la región por parte del gobierno federal y los gobiernos estatales de Oaxaca y Veracruz.

Por otro lado, fue sorprendente la posesión de una hembra juvenil de Tapir Centroamericano (Tapirus bairdii) como mascota, el cual se encontró en perfecto estado de salud, esto se documentó en una ranchería alejada de las zonas urbanas del Municipio de Uxpanapa, Veracruz, por lo que se presentó una controversia: denunciar o no denunciar. Por un lado: 1) se sabe que es una especie en peligro de extinción tanto a nivel nacional; NOM-059-ECOL-2010 (Naranjo 2009), como internacional por la Unión Internacional para la Conservación de la Naturaleza (IUCN; Castellanos 2008), y que su posesión está prohibida; 2) Si se denuncia se estará "rescatando" a este ejemplar pero, la realidad es que se estará condenando a la muerte al ingresarlo a alguno de Los Centros para la Conservación e Investigación de la Vida Silvestre (CIVS) de la Dirección General de Vida Silvestre de la Secretaria Medio Ambiente y Recursos Naturales (SEMARNAT) por la escasa experiencia en mantener este tipo de ejemplares, debido a su carencia de recursos técnicos y económicos; 3) La oportunidad de seguir en la región se nos estaría cerrando; y hasta la integridad física se estaría poniendo en riesgo por llevar a cabo esta denuncia, además de dañar la confianza depositada por el cazador y su familia al mostrarnos este ejemplar; 4) la familia puede quedar sin su pilar económico al ser aprehendido el cazador; y 5) para poder intentar depositarlo a alguna institución zoológica primero hay que decomisarlo y existe una fuerte negativa hacia el personal técnico y autoridades de la Procuraduría Federal de Protección al Ambiente (PROFEPA), ya que se tienen antecedentes y reportes de las comunidades sobre abusos de poder por parte de esta dependencia del Gobierno Federal, por lo que es común que existan conflictos entre las partes. Finalmente, la decisión que se tomó fue charlar con el propietario para que sigan manteniendo al animal, concientizarlo de la importancia que este ejemplar tiene; desde el punto de vista de conservación y el rol de esta especie en los ecosistemas, con la idea final de permitirle que crezca y liberarlo ahí mismo, ya que la ranchería está en el borde de la selva impenetrable de la región. 
Para muchos ambientalistas tal vez esta no sea la mejor decisión, pero se está buscando no perjudicar al comunero, no perder la confianza de los pobladores de la región, liberar al ejemplar una vez adulto y en la misma zona donde fue capturado, además de darle seguimiento desde ese momento hasta su liberación y a posteriori, lo cual aportará al conocimiento de la especie.

Técnicas de cacería utilizadas, perros y armas empleadas. La batida fue la técnica más usada al realizar esta investigación; también se identificaron otras como la espía o acecho en sitios de alimentación y lampareo. Esta técnica aumenta la probabilidad de cazar animales, por lo que debe ser regulada para no poner en riesgo las poblaciones animales sujetas a cacería en la región. El uso de la técnica tiene como ventaja para los cazadores la realización de un esfuerzo mínimo para obtener las presas: mientras mayor sea el número de cazadores que cubran un terreno de caza, más alta es la probabilidad de obtener algún animal; si a esto se agrega el uso de perros, el éxito de caza puede ser aún mayor, pero la desventaja es que la presa se reparte entre los participantes y obtienen menor cantidad de carne. El abuso de la técnica de batida puede traer como consecuencia el agotamiento de las especies aprovechadas. Una incursión con un número alto de cazadores y de perros puede abatir las poblaciones, dejando "limpio" de especies medianas y mayores un terreno de caza. Deben evaluarse alternativas de extracción menos agresivas con el fin de aminorar la presión sobre las poblaciones animales sujetas a la cacería (Quijano-Hernández \& Calmé 2002, Guerra et al. 2010).

Finalmente, la distribución y comercialización de carne de animales silvestres se opera en los restaurantes del Valle de Uxpanapa, Veracruz. El plato de Tepezcuintle (C. paca) frito con ensalada se vende en $\$ 150.00$ por orden en un restaurante típico de la Colonia Cuauhtémoc, Oaxaca. Con esta comercialización es de esperarse que la producción natural de fauna no podrá sostener una extracción tan grande, situación que redunda en la desaparición de las poblaciones de fauna. Aunado a esto, la destrucción de hábitat y ganaderización de la región crean vacíos ecológicos que hacen cada vez más difícil su recuperación en condiciones silvestres (Cid 2001; Reyna \& Tanner 2007).

\section{CONCLUSIONES}

La cacería de subsistencia es una actividad complementaria a las actividades agrícolas y ganaderas que las poblaciones campesinas realizan en la Selva Zoque; sin embargo, es fundamental en el aporte de proteínas de origen animal para las familias más pobres. Las actividades de cacería en la Selva Zoque no se regulan por un plan de manejo, se desarrollan libremente, sin un registro de individuos extraídos, edades y sexos; lo cual puede llevar a un desbalance en las poblaciones. Es necesario continuar realizando monitoreos para planear la sustentabilidad de ese recurso. 
Agradecimientos. Este trabajo no hubiera sido posible sin el apoyo constante de la Congregación de La Fortaleza, Municipio de Santa María Chimalapa, Oaxaca. Agradecemos al Programa Bosques Mexicanos: Selva Zoque, del Fondo Mundial para la Naturaleza (WWF), así como a la Fundación Carlos Slim y la Fundación Comunitaria Oaxaca A.C. por el financiamiento otorgado para la realización del presente estudio. MB-S agradece a la SIP (20090672), Comisión de Operación y Fomento a las actividades Académicas (COFFA) y los Estímulos al Desempeño a la Investigación (EDI), del Instituto Politécnico Nacional (IPN) por el apoyo económico recibido, así como al Sistema Nacional de Investigadores (SNI) por su reconocimiento y apoyo.

\section{LITERATURA CITADA}

Aparicio, R. (Ed.). 2001. Chimalapas. La Última Oportunidad. WWF, SEMARNAP. México.

Arriaga, L., Espinoza, J. M., Aguilar, C., Martínez, E., Gómez, L. \& Loa, E. (Coord.). 2000. Regionesterrestres prioritarias de México. Comisión Nacional para el Conocimiento y Uso de la Biodiversidad, México, D.F.

Ayres, J. M., de Magalhaes Lima, D., de Souza Martins, E. \& Barreiros, J. L. K. 1991. "On the track of the road: changes in subsistence hunting in a Brazilian Amazonian village". Pp. 82-92. In: Robinson, J. G. \& K. H. Redford (Eds). 1991. Neotropical wildlife use and conservation. The University of Chicago Press.

Bello, G. J., \& Reyna-Hurtado, R. 2010. Mazama temama (Kerr 1792), Central America Red rocket Deer, pp. 166-171. In: Barbanti-Duarte, J. M., \& S. Gonzalez (Eds.). Neotropical Cervidology: Biology and medicine of Latin American deer (Jaboticabal, Brazil: Funep and Gland, Switzerland: IUCN.

Bernal, Z. H., Sierra, C., Onaindia, O. M., \& A-Gonzalez, V. T. 2011. Bosques del Mundo; Cambio Climático y Amazonia. Catedra UNESCO-EHU de Desarrollo Sostenible y Educación Ambiental. Keele University.

Bodmer, R. E. \& Robinson, J. G. 2004. Evaluating the sustainability of hunting in the Neotropics, pp. 299-323. In: K.M. Silvius, R.E. Botmer \& J.M.V. Fragoso (Eds.). People in nature: wildlife conservation in South and Central America. Columbia University, Nueva York, USA.

Caballero, J. 2000. Serie de Estudios de Casos del Proyecto de Desarrollo de la Biodiversidad 5. México-Proyecto Reserva Ecológica Campesino, de Los Chimalapas. European Comisión, Deparment for International Development. The Word Conservation Union (IUCN).

Castellanos, A., Foerester, C., Lizcano, D. J., Naranjo, E., Cruz-Aldan, E., Lira-Torres, I., Samudio, R., Matola, S., Schipper, J. \& Gonzalez-Maya. J. 2008. Tapirus bairdii. In: IUCN 2012. IUCN Red List of Threatened Species. Version 2012.2. <www.iucnredlist.org>.

Castro-Franco, R. \& Bustos Z., M.G. 2003. Lagartijas de Morelos, México: distribución, hábitat y conservación. Acta Zoológica Mexicana (n.s.), 88: 123-142.

Cid, I. A. 2001. El aprovechamiento de la fauna silvestre. Pp. 179-188. In: R. Aparicio (Ed.). Chimalapas. La última oportunidad. WWF, SEMARNAP.

CONABIO. 2009. El Capital Natural de México. Vol. I. Conocimiento actual de la biodiversidad. Comisión Nacional para el Conocimiento y Uso de la Biodiversidad. México, D.F., México.

Escamilla, A., Sanvicente, M., Sosa, M. \& Galindo-Leal, C. 2000. Habitat Mosaic, Wildlife Availability, and Hunting in the Tropical Forest of Calakmul, México. Conservation Biology, 14: 15921601.

García, E. 1973. Modificaciones al sistema de clasificación climática de Köppen. Instituto de Geografía, Universidad Nacional Autónoma de México.

Gobierno del Estado de Oaxaca. 1990. Tequio por Chimalapas. Comité Estatal de Planeación para el 
Desarrollo de Oaxaca, Subcomité Especial del COPLADE para la Microrregión de los Chimalapas, Vocalía Ejecutiva de los Chimalapas.

González, M. F. 2004. Las Comunidades Vegetales de México. Secretaría de Medio Ambiente y Recursos Naturales, Instituto Nacional de Ecología. 2a, ed., México, D.F.

González-Pérez, G., Briones-Salas, M. \& Alfaro, A.M. 2004. Integración del conocimiento faunístico del estado. Pp. 449-466. In: A. J. García-Mendoza, M. J. Ordóñez \& M. Briones-Salas (Eds.). Biodiversidad de Oaxaca. Instituto de Biología, UNAM; Fondo Oaxaqueño para la Conservación de la Naturaleza. World Wildlife Fund, México.

Guerra, R. M., Calme, S., Gallina, S. \& Naranjo, E. 2010. Uso y Manejo de la Fauna Silvestre en el Norte de Mesoamérica. Secretaria de Educación, Gobierno del Estado de Veracruz, Xalapa, Veracruz.

Furze, B., de Lacy, T. \& Birckhead, J. 1996. Using methods from the social sciences. In: Culture, conservation and biodiversity. The social dimension of linking local level develop ment and conservation through protected areas. John Wiley \& Sons, West Sussex, Reino Unido.

Laurance W. F. \& Bierregaard Jr., R. O. 1997. Tropical forest remnants: Ecology, management, and communities. University of Chicago Press, Chicago.

Lira-Torres, I. 2006. Abundancia, Densidad, Preferencia de Hábitat y Uso Local de los Vertebrados en Tuza de Monroy, Santiago Jamiltepec, Oaxaca. Revista Mexicana de Mastozoología.10: 6-31.

Lira-Torres, I \& Ramos-Fernández, G. 2007. El Estado del Jaguar en los Chimalapas, Oaxaca. Pp. 71-80. In: G. Ceballos, C. Chávez, R. List, \& H. Zarza (Eds.). Conservación y Manejo del Jaguar en México: Estudios de Caso y Perspectivas. Conabio-Alianza WWF/Telcel-Universidad Nacional Autónoma de México, México D.F.

Lira-Torres, I. \& Briones-Salas, M. 2011. Impacto de la ganadería extensiva y cacería de subsistencia sobre la abundancia relativa de mamíferos en la Selva Zoque, Oaxaca, México. Therya, 2: 217244.

Lira-Torres, I. \& Briones-Salas, M. 2012. Abundancia relativa y patrones de actividad de los mamíferos de los Chimalapas, Oaxaca, México. Acta Zoológica Mexicana (n. s.), 28: 566-585.

Lira-Torres, I., Galindo-Leal, C. \& Briones-Salas, M. 2012. Mamíferos de la Selva Zoque: riqueza, uso y conservación. Revista de Biología Tropical (International Journal of Tropical Biology), 60: 781-797.

Quijano-Hernández, E. \& Calmé, S. 2002.’Patrones de cacería y conservación de fauna silvestre en una comunidad maya de Quintana Roo, México". Etnobiología, 2: 1-18.

Naranjo, E. J., Guerra M., M., Bodmer, R. E. \& Bolaños, J. E. 2004. Subsistence Hunting by Three Ethnic Groups of the Lacandon Forest, México. Journal of Ethnobiology, 24: 233-253.

Naranjo, E. J. 2009. Ecology and Conservation of Baird's tapir in Mexico. Journal-Tropical Conservation Science, 2: 140-158.

Ojasti, J. 2000. Manejo de Fauna Silvestre Neotropical. F. Dallmeir (Ed.). Smithsonian Institution y MAB Program, Washington, D.C., EUA.

Ortega del Valle, D., Carranza, L. T. \& Martínez, J. 2012. Una Mirada desde el Corazón de la Jícara de Oro (Experiencias de Conservación en la Selva Zoque de los Chimalapas). WWF - México / USAID. México, D.F.

Reyna-Hurtado, R. \& Tanner, G. W. 2007. Ungulate relative abundance in hunted and non hunted sites in Calakmul Forest (Southern Mexico). Biodiversity and Conservation, 16: 743-756.

Robinson, J. G. \& Redford, K. H. 1991. Sustainable harvest of Neotropical forest animals, pp. 415429. In: J.G. Robinson and K.H. Redford (Eds.). Neotropical wildlife use and conservation. University of Chicago, Chicago, USA. 
Robinson, J. G. \& Bennett, E. L. (Eds.). 2000. Hunting for sustainability in tropical forest. Columbia University Press, Nueva York, EUA.

Rzedowsky, J. 1991. Vegetación de México. Limusa. México.

Secretaría de Medio Ambiente, Recursos Naturales (SEMARNAT). 2010. Norma Oficial Mexicana NOM-059-ECOL-2010. Protección ambiental, especies de flora y fauna silvestres de México, categorías de riesgo y especificaciones para su inclusión, exclusión o cambio, y lista de especies en riesgo. Diario Oficial de la Federación, Jueves 30 de diciembre de 2010, 1: 1-77.

Salas, M., Schibli, L. \& Torres, B. E. 2001. La importancia ecológica y biológica. Pp. 29-41. In: R. Aparicio (Eds.). Chimalapas. La úlltima oportunidad. WWF, SEMARNAP, México. D.F.

Shaw, J. H. 1991. The outlook for sustainable harvests in Latin America. In: G. Robinson y K. H. Redford (Eds.). Neotropical wildlife use and conservation. (J). University of Chicago Press, Chicago.

Spiegel, M. R., Schiller, J. \& Srinivasan, R. A. 2007. Probabilidad y Estadística. Schaum's Outline of Theory and Problems of Probability and Statistics. Schaum (2 edición). McGraw-Hill. México D.F.

Winter, K. A. 2002. Subsistence use of terrestrial and aquatic animal resources in the tierra comunitaria de origen Itonama of lowland Bolivia. Ph. D. Dissertation, University of Georgia, Athens. 